Supplementary Information for

\title{
Understanding the Role of Self-Adhesion in Crumpling Behaviors of Sheet Macromolecules
}

Yangchao Liao, Zhaofan Li, Sarah Ghazanfari, Fatima,

Andrew B. Croll and Wenjie Xia*

*To whom correspondence should be addressed.

Contact information: wenjie.xia@ndsu.edu

This 4-page SI includes: two tables and one figure

- Table S1

- Table S2

- Figure S1 
Table S1. The Functional Forms and Interaction Parameters of the Coarse-Grained (CG) models of Two-Dimensional (2D) Generalized Macromolecular Sheets. ${ }^{\mathrm{a}}$

\begin{tabular}{llll}
\hline Interaction & Functional forms & Constant parameters & Variable parameters $^{\mathrm{b}}$ \\
\hline Bond & $V_{b}(d)=D_{0}\left[1-\mathrm{e}^{-\alpha\left(d-d_{0}\right)}\right]^{2}$ & $\alpha=1.55 \AA^{-1}$ & $D_{0}=100,196.38,300,350 \mathrm{kcal} / \mathrm{mol}$ \\
& for $d<d_{c u t}$ & $d_{0}=2.8 \AA$ & \\
& & $d_{c u t}=3.25 \AA$ & \\
Angle & $V_{a}(\theta)=k_{\theta}\left(\theta-\theta_{0}\right)^{2}$ & $\theta_{0}=120^{\circ}$ & $k_{\theta}=200,409.40,500,700 \mathrm{kcal} / \mathrm{mol}$ \\
Dihedral & $V_{d}(\phi)=k_{\phi}[1-\cos (2 \phi)]$ & - & $k_{\phi}=0,4.15,10,15 \mathrm{kcal} / \mathrm{mol}$ \\
Non-bonded & $V_{n b}\left(r_{L J}\right)=4 \varepsilon_{L J}\left[\left(\frac{\sigma_{L J}}{r_{L J}}\right)^{12}-\left(\frac{\sigma_{L J}}{r_{L J}}\right)^{6}\right]$ & $\sigma_{L J}=3.46 \AA$ & $\varepsilon_{L J}=0.30 .82,3,5 \mathrm{kcal} / \mathrm{mol}$ \\
& for $r_{L J}<r_{c u t}$ & & \\
& & &
\end{tabular}

$\overline{{ }^{a}}$ The molecular dynamics model of 2D macromolecular sheet is based on the coarse-grained graphene model developed using the strain energy conservation method. ${ }^{1}$

${ }^{b}$ The values of variable parameters were chosen to obtain models with varying properties.

Table S2. The Obtained Values of $a_{g}, a_{h}, m_{g}$, and $m_{h}$ of the Size Scaling Relationships for Different $\varepsilon_{L J}$ from the Simulations. ${ }^{\mathrm{c}}$

\begin{tabular}{lllll}
\hline$\varepsilon_{L J}(\mathrm{kcal} / \mathrm{mol})$ & $a_{g}$ & $m_{g}$ & $a_{h}$ & $m_{h}$ \\
\hline 0.3 & $0.887 \pm 0.005$ & $0.928 \pm 0.002$ & $1.548 \pm 0.078$ & $0.763 \pm 0.014$ \\
0.82 & $0.835 \pm 0.005$ & $0.943 \pm 0.002$ & $1.403 \pm 0.068$ & $0.787 \pm 0.014$ \\
3 & $0.733 \pm 0.006$ & $0.974 \pm 0.002$ & $1.094 \pm 0.061$ & $0.845 \pm 0.017$ \\
5 & $0.699 \pm 0.006$ & $0.984 \pm 0.002$ & $0.996 \pm 0.062$ & $0.866 \pm 0.017$ \\
\hline
\end{tabular}

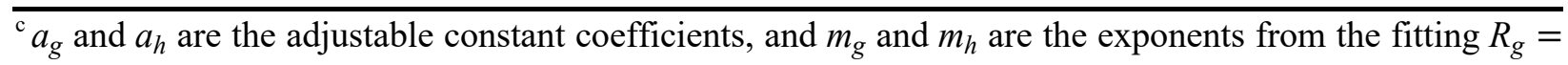
$a_{g} R_{c}^{m_{g}}$ and $R_{h}=a_{h} R_{c}^{m_{h}}$, respectively. 


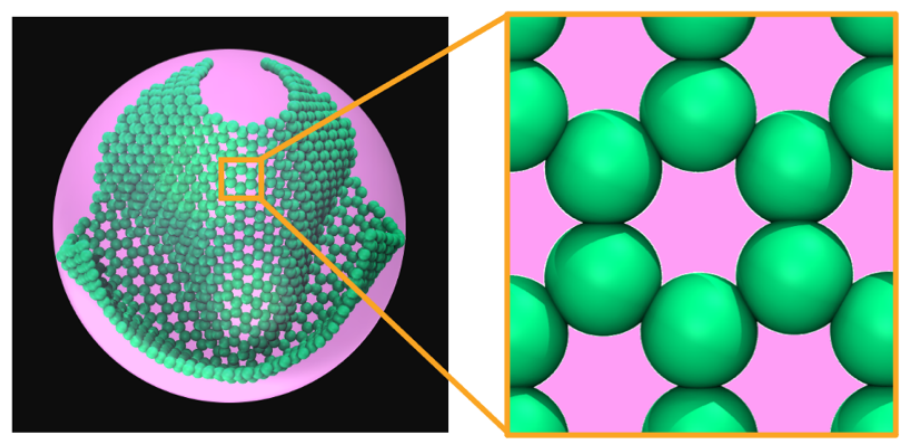

Figure S1. A schematic representation of the computational principle of crumpled macromolecular sheet in ZENO software.

In Figure S1, each bead of the macromolecular sheet model is represented by a sphere with radius of $1.73 \AA$ (i.e., half of $\sigma_{L J}, \sigma_{L J}=3.46 \AA$ is the parameter of the function for non-bonded interaction as shown in Table $\mathrm{S} 1$ ). To calculate the hydrodynamics radius $R_{h}$ of the crumpled macromolecular sheet, a launching sphere (pink sphere) is created around a crumpled macromolecular sheet (green model), and then random walks are initiated starting with the surface of the launching sphere. Two possibilities for these random walks are either to hit the crumpled sheet or to return to the launching sphere. Given the probability in the second case, the walks may either reach infinity or re-launch in order to detect the sheet again. In present study, the random walks for ZENO path-integral calculation is $1 \times 10^{5}$. Specifically, the hydrodynamics radius $R_{h}$ is calculated in ZENO following the equation below ${ }^{2}$ :

$$
R_{h}=q_{R_{h}} t R
$$

where $q_{R_{h}} \approx 1$ is the prefactor, $t$ is the fraction of random walks that hit the sheet as opposed to go to infinity, and $R$ is the radius of the launching sphere. 


\section{References}

1. Ruiz, L., Xia, W., Meng, Z. \& Keten, S. A coarse-grained model for the mechanical behavior of multi-layer graphene. Carbon 82, 103-115 (2015).

2. Juba, D., Audus, D. J., Mascagni, M., Douglas, J. F. \& Keyrouz, W. ZENO: Software for calculating hydrodynamic, electrical, and shape properties of polymer and particle suspensions. J. Res. Natl. Inst. Stand. Technol. 122, 1-2 (2017). 